\title{
A Criterion for Quasi-Steadiness of the Laminar Diffusion Flamelet Model
}

\author{
B. $\mathrm{Hu}^{1 \dagger}$ and C. J. Rutland ${ }^{2}$ \\ ${ }^{1}$ Cummins Inc., Columbus, Indiana, ,USA \\ ${ }^{2}$ University of Wisconsin-Madison, Madison, Wisconsin, USA \\ †Corresponding Author Email: bing.hu@cummins.com
}

(Received January 10, 2013; accepted April 3, 2013)

\begin{abstract}
The laminar diffusion flamelet model is a well-established approach to modeling turbulent non-premixed combustion. Its quasi-steady version, referred to as the steady flamelet model, is very useful in engineering CFD calculations due to its low computational cost. However, previous studies have shown that unsteadiness of flamelets needs to be taken into account under certain circumstances. In this study, we derive a flamelet time scale, which is a characteristic time period needed for a flamelet structure to reach its quasi-steady state, and we use this time scale to propose a criterion to test quasi-steadiness of the flamelet. Although this criterion is rather conservative in that the predicted steadyflamelet regime tends to be smaller than it should be, the criterion is easy to use because it only requires the information from the mixing field.
\end{abstract}

Keywords: Turbulent non-premixed combustion, Modeling, Flamelet time scale, Scalar dissipation rate.

\section{NOMENCLATURE}

\author{
$Y_{i} \quad$ mass fraction of species $i$ \\ $\mathrm{Z} \quad$ mixture fraction \\ $\chi \quad$ scalar dissipation rate \\ $(\Delta Z)_{D}$ diffusion zone thickness in a flamelet
}

\section{INTRODUCTION}

The laminar diffusion flamelet model is a wellestablished approach to modeling turbulent nonpremixed combustion, (Peters 1984). Its quasi-steady version, referred to as the steady flamelet model or the stationary laminar flamelet model, is based on the view that the flamelet structure can instantaneously reach its quasi-steady state, so that unsteady effects are negligible. Thanks to this quasi-steadiness, the flamelet calculations can be completely decoupled from the CFD calculations. The common practice is to perform flamelet calculations as a pre-processing step and store the steady flamelet solutions in a library, which are looked up during the later CFD calculations (Lee and Rutland 2002; $\mathrm{Hu}$ et al. 2010). The steady flamelet model has been shown both efficient and effective in engineering calculations (Lee and Rutland 2002). However, it has also been shown that unsteady effects must be taken into account in those situations discussed in (Haworth et al. 1988; Barlow and Chen 1992; Pitsch

$\begin{array}{ll}(\Delta Z)_{R} & \text { reaction zone thickness in a flamelet } \\ \tau_{\eta} & \text { kolmogrov time scale } \\ \tau_{f l a m e l e t} & \text { flamelet time scale } \\ \omega_{i} & \text { chemical reaction rate of species } i\end{array}$

and Peters 1998; Cuenot et al. 2000). All this begs the question: Under what conditions can a laminar flamelet structure be assumed to be quasi-steady?

Mell et al. (1994) and Swaminathan (2002) have independently proposed the criteria for testing quasisteadiness of the laminar flamelet model. Although these criteria are useful in theoretical analysis or direct numerical simulations (DNS), they are difficult to use in practical combustion modeling. This is because they need the time scale information about both flow motions and chemical reactions. In particular, obtaining an accurate chemical time scale is difficult for nonpremixed combustion in practical calculations. In the present study, we have developed a criterion that can be used to distinguish the steady flamelet regime from the unsteady flamelet regime, and this criterion does not need information about chemical reactions. 


\section{LAMINAR DIFFUSION FLAMELET MODEL}

The laminar flamelet model is based on the flamelet assumption, which is valid when the mixing field is locally one-dimensional (Mell et al. 1994). Thus, in a turbulent diffusion flame, the reaction zone is assumed to be locally like a one-dimensional laminar flame embedded in the turbulent flow field. Mathematically, the transport equation of a reactive scalar can be simplified by taking advantage of the onedimensionality of the reaction zone. Following Peters (1984), the conservation equation of a reactive scalar can be transformed from the physical space $\left(x_{1}, x_{2}, x_{3}\right)$ into a local mixture fraction space $\left(Z, Z_{2}, Z_{3}\right)$. Here, $Z$ represents the mixture fraction coordinate normal to the primary scalar iso-surface, $Z_{2}$ and $Z_{3}$ represent the orthogonal coordinates perpendicular to the $Z$-direction. The gradients in the $Z_{2^{-}}$and $Z_{2^{-}}$directions are of lower order compared to those in the $Z$ - direction and thus can be neglected. The simplified transport equation is called the flamelet equation, which, for the chemical species $i$, can be written as

$\frac{\partial Y_{i}}{\partial t}=\frac{\chi}{2} \frac{\partial^{2} Y_{i}}{\partial Z^{2}}+\frac{\omega_{i}}{\rho}$.

Here, $Y_{i}$ is the mass fraction of species $i, \rho$ is the density, $t$ is time, $Z$ is mixture fraction, $\omega_{i}$ is the chemical reaction rate of species $i$, and $\chi$ is the scalar dissipation rate, defined as

$\chi=2 D \frac{\partial Y_{i}}{\partial x_{k}} \frac{\partial Y_{i}}{\partial x_{k}}$,

where $D$ is the diffusivity of mixture fraction and $x_{k}$ is the physical coordinate in index notation.

\section{Flamelet Time Scale}

We define the flamelet time scale as a characteristic time period that the unsteady term, $\partial Y_{i} / \partial t$, in Eq. (1) takes to vanish. In other words, it is the time length needed for a flamelet structure to reach its quasi-steady state. Following this line of thought, the flamelet time scale can be directly derived from the flamelet equation.

Although the flamelet equation is consistently valid in the entire space of mixture fraction, for the sake of argument we split the mixture fraction space into two zones: a diffusion zone and a reaction zone. Typically, the reaction zone corresponds to the thin region adjacent to the stoichiometric mixture fraction and the diffusion zone corresponds to the rest of the domain. Following the terminologies of boundary layer theory, the diffusion zone and the reaction zone can be also called the outer layer and inner layer, respectively.

In the outer layer (the diffusion zone), chemical reactions are negligible. Hence, the flamelet equation is simplified to

$\frac{\partial Y_{i}}{\partial t}=\frac{\chi}{2} \frac{\partial^{2} Y_{i}}{\partial Z^{2}}$

Properly scaling each term shows $\frac{\partial Y_{i}}{\partial t} \sim \frac{\left(\Delta Y_{i}\right)_{D}}{\tau_{o}}$,

$\frac{\chi}{2} \frac{\partial^{2} Y_{i}}{\partial Z^{2}} \sim \frac{\chi}{2} \frac{\left(\Delta Y_{i}\right)_{D}}{(\Delta Z)_{D}^{2}}$

where $\left(\Delta Y_{i}\right)_{D}$ is the characteristic scale of $Y_{i}$ in the diffusion zone, $\tau_{o}$ is the time scale in the diffusion zone (i.e., the outer layer time scale), and $(\Delta Z)_{D}$ is the diffusion zone thickness measured in mixture fraction. Substituting these scales into Eq. (3) and solving for $\tau_{o}$ gives

$\tau_{o} \sim \frac{2(\Delta Z)_{D}^{2}}{\chi}$.

In the inner layer (the reaction zone), there must be only three scenarios in terms of the relative importance of mixing and chemistry:

Case (I): The mixing is much faster than the chemical reactions. This is relevant when flame extinction occurs. In this case, the reaction term is negligible compared to the mixing term in the inner layer. Hence, the unsteady term can be approximated by the mixing term alone:

$\frac{\partial Y_{i}}{\partial t} \approx \frac{\chi}{2} \frac{\partial^{2} Y_{i}}{\partial Z^{2}}$.

Following the same reasoning that leads to Eq. (6), we obtain the inner time scale for this case:

$\tau_{i}^{I} \sim \frac{2(\Delta Z)_{R}^{2}}{\chi}$,

where $(\Delta Z)_{R}$ is the reaction zone thickness measured in mixture fraction. Note that $\tau_{i}^{I}$ in Eq. (8) is similar to $\tau_{o}$ in Eq. (6) except for the different $\Delta Z$ being used.

Case (II): The mixing is much slower than the chemical reactions. This is relevant in homogeneous-like combustion, which involves a near-zero scalar dissipation rate. In this case, the unsteady term only depends on the chemical term, and the inner time scale is estimated as:

$\tau_{i}^{I I} \sim \tau_{\text {chem }} \mid(\chi \approx 0)$,

where $T_{\text {chem }} \mid(X \approx 0)$ represents the chemical time scale given a near-zero scalar dissipation rate.

Case (III): The mixing is comparable to the chemical reactions. This is typical in mixing-controlled combustion: The speed of mixing determines the speed of chemical reactions; the mixing term and reaction term in the flamelet equation tend to balance each other. Hence, the inner flamelet time scale approaches zero:

$\tau_{i}^{I I I} \approx 0$.

Since the flamelet time scale is defined as the time needed for the unsteady term in the flamelet equation to vanish, a general flamelet time scale should be chosen as the largest one among all the possible scenarios above, i.e., 
$\tau_{\text {flamelet }}=\max \left(\tau_{o,} \tau_{i}^{I}, \tau_{i}^{I I}, \tau_{i}^{I I I}\right)$.

Substituting for $\tau_{i}^{I}, \tau_{i}^{I I}$, and $\tau_{i}^{I I I}$ using Eqs (8), (9), (10) respectively, we obtain:

$\tau_{\text {flamelet }}=\max \left(\frac{2(\Delta Z)_{D}^{2}}{\chi}, \frac{2(\Delta Z)_{R}^{2}}{\chi}, \tau_{\text {chem }}, \mid(\chi \approx 0), 0\right)$

In Eq. (12), the last two terms are inevitably smaller than the first two for two reasons. First, the first two terms are positive definite. Second, the third term becomes relevant only in case that scalar dissipation rate is near-zero; in this case, either of the first two terms, which is divided by $\chi$, must be larger than the third term. Hence, Eq. (12) can be simplified to

$$
\tau_{\text {flamelet }}=\max \left(\frac{2(\Delta Z)_{D}^{2}}{\chi}, \frac{2(\Delta Z)_{R}^{2}}{\chi}\right)
$$

Pitsch et al. (1998) proposed a characteristic diffusion time $t_{\gamma}$ which it takes to transport mass and energy over a distance $\Delta Z$ (Pitsch et al. 1998):

$t_{\chi}=\frac{(\Delta Z)_{D}^{2}}{\chi_{s t}}$,

where $\Delta Z$ is the reaction zone thickness and $\chi_{s t}$ is the scalar dissipation rate evaluated at the stoichiometric condition. Within the current context, this characteristic time can be also interpreted as the flamelet time scale in the reaction zone. Compared to Pitsch's time scale, the flamelet time scale proposed here is an improvement in that it accounts for the flamelet behavior in both the reaction zone and diffusion zone.

\section{CRITERION OF QUASI-STEADINESS}

The quasi-steadiness of a flamelet can be justified only if the associated flamelet time scale is short compared to the external time scale:

$\tau_{\text {flamelet }}<\tau_{\text {external }}$.

Now the question becomes how to determine the external time scale. In modeling a turbulent diffusion jet flame, Pitsch et al. (1998) proposed a Lagrangian flamelet time as

$t=\int_{0}^{x} \frac{1}{u\left(x^{\prime}\right) \mid\left(Z=Z_{s t}\right)} d x^{\prime}$,

where $x$ is the axial coordinate, $Z$ is mixture fraction and $u\left(x^{\prime}\right) \mid\left(Z=Z_{s t}\right)$ is the axial velocity component at the radial position where $Z=Z_{s t}$. This is a Lagrangian flamelet time and can be interpreted as the flamelet life time. Hence, it is a suitable choice for the external time scale in a simple jet flame.

Here, we suggest a more general external time scale based on the following reasoning. The only external parameter in the flamelet equation is the scalar dissipation rate, which parameterizes the external influence from the flow field. Therefore, an appropriate choice of the external time scale is the evolution time scale of the scalar dissipation rate, which is denoted by $\tau_{\chi}$ and defined as $\tau_{\chi}=\frac{\chi}{|\dot{\chi}|}$

The ratio $\chi /|\dot{\chi}|$ has been shown to be proportional to the Kolmogrov time scale: $\chi /|\dot{\chi}| \approx 4 \tau_{\eta}$ in Yeung et al. (1990). Substituting this finding into Eq. (17) gives

$\tau_{\chi} \approx 4 \tau_{\eta}$.

Substituting Eq. (13) for $\tau_{\text {flamelet }}$ and substituting Eq. (18) for $\tau_{\text {external }}$ into the criterion Eq. (15), we obtain a criterion to test quasi-steadiness of a laminar diffusion flamelet:

$\max \left(\frac{2(\Delta Z)_{D}^{2}}{\chi}, \frac{2(\Delta Z)_{R}^{2}}{\chi}\right)<2 \tau_{\eta}$.

This criterion is referred to as the "strong" criterion here. It needs information about not only mixing, included in $\chi$ and $(\Delta Z)_{D}$, but also chemistry, included in $(\Delta Z)_{R}$. This criterion is useful in DNS studies when thickness of both the reaction zone and diffusion zone can be readily available. However, in engineering calculations, such as RANS and LES, these values are difficult to obtain. To make it easier to use, a simplification about this criterion can be made based on the following reasoning.

Under a high Damköhler number condition, we have $\max \left(2(\Delta Z)_{D}^{2} / \chi, 2(\Delta Z)_{R}^{2} / \chi\right)=2(\Delta Z)_{D}^{2} / \chi$ due to thinness of the reaction zone. However, under a low Damköhler number condition, the reaction zone thickness can be comparable or even larger than the diffusion zone thickness, so that:

$\max \left(2(\Delta Z)_{D}^{2} / \chi, 2(\Delta Z)_{R}^{2} / \chi\right)=2(\Delta Z)_{R}^{2} / \chi$.

In both cases, the upper bound of both $(\Delta Z)_{D}$ and $(\Delta Z)_{R}$ is unity. Therefore, using this upper bound, we obtain a "weak" but more practical criterion for quasisteadiness:

$\chi>\frac{1}{2 \tau_{\eta}}$.

Note that because of the upper bound of $\Delta Z$ being used, the weak criterion is rather conservative, which means that it tends to predict a smaller steady-flamelet regime than the one predicted by the strong criterion, Eq. (19). However, the advantage of the weak criterion is that it only requires the information from the mixing field, represented by $\chi$.

\section{CONClusion}

We have derived a flamelet time scale, Eq. (13), and a new criterion for testing quasi-steadiness of a laminar diffusion flamelet, Eq. (19) or Eq. (20). The flamelet time scale can be used to modify a steady flamelet solution to incorporate unsteady effects in the means proposed by Rao et al. (2003). The criterion, Eq. (19) or Eq. (20), can be used to define the boundary between the steady flamelet regime and the unsteady flamelet regime in a multi-mode combustion model (Hu et al. 2010) or a hybrid unsteady/steady flamelet model. The boundary suggested by the weak criterion, Eq. (20), tends to shift toward the steady-flamelet regime. But in terms of model accuracy, this should not be a problem 
because an unsteady-flamelet model can automatically adapt itself to the steady state in the steady-flamelet regime.

\section{REFERENCES}

Barlow, R. and J. Chen (1992). On transient flamelets and their relationship to turbulent methane-air jet flames. Proceedings of the Combustion Institute, 231-237.

Cuenot, B., F. Egolfopoulos, and T. Poinsot (2000). An unsteady laminar flamelet model for non-premixed combustion. Combustion Theory and Modeling, 4, 77-97.

Haworth, D., D. Drake, S. Pope, and R. Blint (1988). Unsteady flamelet modeling of turbulent hydrogenair diffusion flames. Proceedings of Combustion Institute, 1057-1064.

Hu, B., T. Shethaji, and C.J. Rutland (2010). A mixedmode combustion model for large-eddy simulation of diesel engines. Combustion Science and Technology ,182, 1279-1320.

Lee, D. and C.J. Rutland (2002). Probability density function combustion modeling of diesel engines. Combustion Science and Technology, 174, 19-54.
Mell, W., V. Nilsen, G. Kosály, and J. Riley (1994). Investigation of closure models for nonpremixed turbulence reacting flows. Physics of Fluids, 6, 1331-1356.

Peters, N. (1984). Laminar diffusion flamelet models in non-premixed turbulent combustion. Progress in Energy and Combustion Science, 10(3), 319-339.

Pitsch, H. and N. Peters (1998). Unsteady flamelet modeling of turbulent hydrogen-air diffusion flames. Proceedings of the Combustion Institute, 1057-1064.

Rao, S. and C. Rutland (2003). A flamelet time scale model for non-premixed combustion including chemical kinetic effects. Combustion and Flame, 133, 189-191.

Swaminathan, N. (2002). Flamelet regime in nonpremixed combustion. Combustion and Flame, 129, 217-219.

Yeung, P., S. Girimaji, and S. Pope (1990). Straining and scalar dissipation on material surfaces in turbulence: Implications for flamelets. Combustion and Flame, 79, 340-365. 